\title{
Experimental Design and Inverse Problems in Plant Biological Modeling
}

\author{
M. Avery, H.T. Banks ${ }^{\dagger}$ K. Basu, Y. Cheng $§$ \\ E. Eager, ${ }^{\llbracket}$ S. Khasawinah, L. Potter, K.L. Rehm ${ }^{\dagger \dagger}$
}

December 5, 2011

\begin{abstract}
We develop a mathematical and statistical framework to model the actions of underlying metabolites for carbon dioxide assimilation in photosynthesis. This study was motivated by a challenge posed by Syngenta Biotechnology to use modeling to better characterize photosynthesis in plants and subsequently better understand growth and crop yield. We use a dynamical system model proposed by Zhu., et al. [16], which describes the Calvin Cycle in spinach plants through changes in the concentrations of 38 metabolites (state variables) using non-linear enzyme kinetic ordinary differential equations and mass-balance laws that contain a total of 165 parameters. In our study of the $\mathrm{CO}_{2}$ assimilation rate, we pose our research questions with this dynamical system mathematical model and a statistical model to describe the observation process. In particular, we address the research question "Once a subset of parameters is fixed and the times at which data is collected are determined, can we identify which metabolites we should measure in order to optimize the confidence in our parameter estimates?" This problem of choosing the best subset of metabolites to measure is not well-explored in the existing literature. Here, we propose two methods. The first, the ad hoc Simple Linear Regression (SLR) method, models carbon assimilation as a linear function of the metabolites. Using simulated data, we implement a step-wise selection algorithm to determine the best subset of metabolites at each of 10 fixed time points. The second method, the Optimal Design Criterion method, fixes the number of metabolites to be observed and searches for the best combination by calculating a measure of the size of the Fisher Information Matrix (FIM) associated with measuring only the selected metabolites. Both methods suggest promise in determining appropriate sets of metabolites to observe for successful implementation of inverse problems. Our conclusions represent a paradigm for estimating parameters and designing experiments more efficiently in plant biological modeling. Ultimately, our results may be used to help engineer seeds that maximize carbon dioxide assimilation in photosynthesis and hence promote plant growth.
\end{abstract}

\section{Introduction and Motivation}

In today's world, the demand for more food and energy is skyrocketing while resources are rapidly declining. The population is growing, urbanization is continuing, diets are changing, and our need for more energy as fuel is rising; however, we do not have the land or water resources to sustain this inevitable modernization. Agriculture already uses $40 \%$ of the world's land surface and $70 \%$ of all available freshwater. Caught in this paradox of producing enough food and fuel for the world while resources are running out, we need to think outside of the box. Mathematics and statistics provide a means towards better understanding these problems and a path towards solving them in a more efficient way.

Currently, biomass production is related to available resources in sophisticated crop models, such as the Aquacrop model [15] and decision support system for agrotechnology transfer (DSSAT) [13]. Standard approaches in crop modeling relate crop yields or biomass production to a plethora of environmental factors such as water, sunlight, soil nutrients,

\footnotetext{
${ }^{*}$ Department of Statistics, North Carolina State University

${ }^{\dagger}$ Center for Research in Scientific Computation and Department of Mathematics, North Carolina State University

$\ddagger$ Department of Mathematics, University of South Carolina

$\S$ Department of Statistics, Boston University

『 Department of Mathematics, University of Nebraska-Lincoln

" Department of Statistics, Johns Hopkins University

**Bioinformatics, Syngenta Biotechnology, Inc., Research Triangle Park, NC

${ }^{\dagger}$ Center for Research in Scientific Computation, and Department of Mathematics, North Carolina State University
} 
and crop management practices. These models do not include compartments for the enzyme processes that occur in plants. The relatively simple Aquacrop model [15] utilizes basic mathematics and patterns observed in plant growth to mainly relate plant growth to water consumption using the formula $B=W P \times \sum T r$, where $B$ is biomass, $W P$ is water productivity of the crop, and $T r$ is crop transpiration. The DSSAT utilizes a modular algorithm and includes many more factors in plant growth but has only been tested and verified on about 16 crops in its history from 1988 to 2003. It utilizes the CROPGRO algorithm, which was originally developed for soybean and peanut growth but has been successfully extended to wheat. Both systems are useful for predicting overall crop yields; however, they cannot be fine-tuned for changes in enzyme reaction rates caused by genetically modifying seeds.

In [12], the authors present a simple mathematical model (compared to that of [16]) for calculating carbon dioxide assimilation in the Calvin Cycle. This nonlinear ordinary differential equation (ODE) model uses atmospheric $\mathrm{CO}_{2}$ and $\mathrm{O}_{2}$ concentrations, light intensity, temperature and some composite reaction rates to describe $\mathrm{CO}_{2}$ assimilation and the changes in concentration of NADPH, NADP ${ }^{+}$, ATP, ADP, PGA, and RUBP. The model is developed at the cellular level and extended to the leaf level, where light intensity has a more pronounced and nonlinear effect. The model draws on previous knowledge of reactions in leafy plants such as spinach and eucalyptus. The techniques used to develop this model may be useful if we were to add more environmental factors to the model in [16] without moving to a crop modeling system.

There are many techniques that may be used to inform the design of experiments. We focus on nonlinear least squares methods. In [3], the methodologies of maximum likelihood estimators (MLE), ordinary least squares (OLS), and generalized least squares (GLS) are discussed with scalar and vector observations, and uncertainty quantification is mentioned, namely in the form of observation error covariances, standard errors, and, for scalar data, sensitivity matrices. A related metric of uncertainty in parameter estimates known as the Fisher Information Matrix (FIM) is then explained and used to identify optimal sampling times for ODE systems with scalar and vector observations in [8]. Experimental design using sensitivity functions as well as the FIM is described in [7].

The question of what variables to measure is very important in the context of this and many other plant enzyme kinetics models. It is always preferable to reduce the time and cost required to sample specimens while maximizing the useful information from the collected data, and it is highly unlikely that observations of only one metabolite would provide sufficient information to estimate parameters. However, how should one choose this one metabolite if only one is available?

In this paper we analyze the interactions of state variables and parameters in a mathematical and statistical model of carbon dioxide assimilation in the Calvin Cycle for spinach to determine which metabolites should be measured in an experiment. Our model consists of a system of coupled differential equations describing the relationship between the metabolites involved in the Calvin Cycle [16] and a corresponding inverse problem framework utilizing the statistical model to determine optimal parameter estimates and experimental design.

Our results illustrate that by modifying the ratios of the concentrations of enzymes involved in carbon metabolism or the parameters describing the efficiency of the reactions that use these metabolites, the photosynthetic rate can be changed. In particular, the model output is most sensitive to a few parameters. In addition to aforementioned inverse problem approach, we also develop an innovative algorithm to determine which metabolites are best to measure. These findings in optimal experimental design and parameter estimation theory in plant biological modeling could help to develop more efficient seeds to yield better results despite diminishing resources.

\section{Biology Background}

Photosynthesis is the process by which plants convert solar energy, using carbon dioxide and water, into chemical energy and byproducts. The plant stores the chemical energy in the form of glucose and uses this carbohydrate energy source to grow. The chemical equation for photosynthesis in plants is:

$$
6 \mathrm{CO}_{2}+\mathrm{H}_{2} \mathrm{O}=\mathrm{C}_{6} \mathrm{H}_{12} \mathrm{O}_{6}+6 \mathrm{CO}_{2}+6 \mathrm{H}_{2} \mathrm{O} .
$$

Photosynthesis takes place in the chloroplasts and involves two stages. The energy transferring stage, sometimes called the light-dependent reactions, occurs in cell units called photosystems located in the thylakoid membranes of chloroplasts. In this stage, pigment molecules capture energy (in the form of photons) from the sun and store it temporarily in the chemical bonds of adenosine triphosphate (ATP) and reduced nicotinamide adenine dinucleotide phosphate (NADPH), which is an electron carrier. In this process, one molecule of the pigment chlorophyll absorbs one photon and 
loses one electron. Then, in a process called photolysis, the chlorophyll molecule uses water to regain the lost electron, which releases oxygen.

In the carbon fixation stage, ATP and NADPH are used to repackage the energy as sugar and starch. There are several common pathways used by plants to perform carbon fixation. Spinach utilizes the $\mathrm{C} 3$ pathway, also known as the the Calvin Cycle or light-independent reactions. The Calvin Cycle is an intricate series of reactions that can be categorized into three stages. In the first stage, the energy in ATP is used to bind the sugar ribulose 1,5-bisphosphate (RuBP) to $\mathrm{CO}_{2}$ to create two molecules of 3-phosphoglycerate (PGA). This stage utilizes the enzyme known as RuBisCO. In the next stage, the energy in NADPH is used to reduce PGA to 3-phoshoglyceraldehyde (PGAL) in reactions catalyzed by the enzymes phosphoglycerate kinase and G3P dehydrogenase. This stage also produces nicotinamide adenine dinucleotide phosphate $\left(\mathrm{NADP}^{+}\right)$and adenosine diphosphate (ADP), which are then used in the energy transferring stage to create NADPH and ATP again. Finally, in the regeneration stage, most of the PGAL is rearranged to recreate RuBP. A portion of the PGAL moves into the cytosol to be used in the creation of sucrose.

Also included in this model of the Calvin Cycle as performed in a spinach leaf [16] is the mechanism of photorespiration that occurs in many plants in bright light. The enzyme RuBisCO catalyzes a reaction that uses some of the newly produced sugars to change $\mathrm{O}_{2}$ into $\mathrm{CO}_{2}$. These reactions reduce the efficiency of photosynthesis and are important to include when looking at the large system of enzyme kinetic reactions that occur in a plant.

This model is for the Calvin Cycle of a spinach leaf [16], though this prototype could be easily generalized to other plants. We use both statistics and applied mathematics to formulate an inverse problem. Because the observation process inherently contains errors, which affect resultant parameter estimates, and the system is naturally dynamic, the model will involve a system of coupled ordinary differential equations and a corresponding statistical model to characterize the errors in measurement. We solve this model using parameter estimation theory [3, 14].

\section{Modeling Techniques}

\subsection{Mathematical and statistical models}

Solving this problem requires both a mathematical and a statistical model, which we define below. The mathematical model is given by

$$
\begin{aligned}
\frac{d \vec{x}}{d t}(t) & =\vec{g}(t, \vec{x}(t ; \vec{\theta}), \vec{q}), \quad t \in\left[t_{0}, t_{f}\right] \\
\vec{x}\left(t_{0} ; \vec{\theta}\right) & =\vec{x}_{0}
\end{aligned}
$$

where $\vec{x}(t ; \vec{\theta})$ is the vector of state variables of the system generated using parameter vector $\vec{\theta}=\left(\vec{x}_{0}, \vec{q}\right) \in \mathbb{R}^{p}, p=m+r$, that contains $m$ initial values and $r$ system parameters listed in $\vec{q}, \vec{g}$ is a mapping $\mathbb{R}^{1+m+r} \rightarrow \mathbb{R}^{m}, t_{0}$ is the initial time, and $t_{f}$ is the final time. We define an observation process

$$
\vec{f}(t ; \vec{\theta})=\mathcal{C} \vec{x}(t ; \vec{\theta}),
$$

where $\mathcal{C}$ is an observation operator that maps $\mathbb{R}^{m} \rightarrow \mathbb{R}^{N}$. If we were able to observe all variables, then $\mathcal{C}=\mathbf{I}^{m \times m}$ and $N=m$; however, this is often not the case because it is impossible or too expensive to measure all state variables.

In order to discuss the amount of uncertainty in parameter estimates, we formulate a statistical model [3] of the form

$$
\vec{Y}(t)=\vec{f}\left(t ; \vec{\theta}_{0}\right)+\overrightarrow{\mathcal{E}}(t), \quad t \in\left[t_{0}, t_{f}\right]
$$

where $\vec{\theta}_{0}$ are the (standard for statistical treatments) hypothesized true values of the unknown parameters and $\overrightarrow{\mathcal{E}}$ is a vector of random variables that represents observation error for the state variables and follows the following assumptions

$$
\begin{aligned}
\mathrm{E}(\overrightarrow{\mathcal{E}}(t)) & =\overrightarrow{0}, \quad t \in\left[t_{0}, t_{f}\right], \\
\operatorname{Var}(\overrightarrow{\mathcal{E}}(t)) & =V_{0}(t)=\operatorname{diag}\left(\sigma_{0,1}(t)^{2}, \sigma_{0,2}(t)^{2}, \ldots, \sigma_{0, N}(t)^{2}\right), \quad t \in\left[t_{0}, t_{f}\right], \\
\operatorname{Cov}\left(\mathcal{E}_{i}(t) \mathcal{E}_{i}(s)\right) & =\sigma_{0, i}(t)^{2} \delta(t-s), \quad s, t \in\left[t_{0}, t_{f}\right], \\
\operatorname{Cov}\left(\mathcal{E}_{i}(t) \mathcal{E}_{j}(s)\right) & =0, \quad i \neq j, \quad s, t \in\left[t_{0}, t_{f}\right],
\end{aligned}
$$


where $\delta(0)=1$ and $\delta(t)=0$ for $t \neq 0$. One realization of the statistical model (3) is written

$$
\vec{y}(t)=\vec{f}\left(t, \overrightarrow{\theta_{0}}\right)+\vec{\epsilon}(t), \quad t \in\left[t_{0}, t_{f}\right] .
$$

When collecting experimental data, it is often difficult to take continuous measurements of the observed variables. Instead, we assume that we have $n$ observations at times $t_{j}, j=1, \ldots, n, t_{0} \leq t_{1}<t_{2}<\ldots<t_{n} \leq t_{f}$. We write the observation process (2) as

$$
\vec{f}\left(t_{j} ; \vec{\theta}\right)=\mathcal{C} \vec{x}\left(t_{j} ; \vec{\theta}\right), \quad j=1,2, \ldots, n,
$$

the discrete statistical model as

$$
\vec{Y}_{j}=\vec{f}\left(t_{j} ; \vec{\theta}_{0}\right)+\overrightarrow{\mathcal{E}}\left(t_{j}\right), \quad j=1,2, \ldots, n,
$$

and a realization of the discrete statistical model as

$$
\vec{y}_{j}=\vec{f}\left(t_{j}, \vec{\theta}_{0}\right)+\vec{\epsilon}\left(t_{j}\right), \quad j=1,2, \ldots, n .
$$

If we were given $\vec{\theta}$, we could solve (1) for $\vec{x}(t ; \vec{\theta})$, a process known as performing the forward problem. Alternatively, if we had a set of data $\vec{y}_{j}, j=1,2, \ldots, n$, we could estimate $\vec{\theta}_{0}$ in a process known as solving the inverse problem. We intend to use this mathematical and statistical framework to determine which variables would provide the most useful information if measured, when those variables should be measured, and what parameters we can most confidently estimate.

\subsection{Ordinary least squares}

Several methods exist to solve the inverse problem [3]. A major factor in determining which method to use is additional assumptions made about $\overrightarrow{\mathcal{E}}(t)$. It is common practice to make the assumption that realizations of $\overrightarrow{\mathcal{E}}(t)$ at particular time points are independent and identically distributed (i.i.d.). If, additionally, the distributions describing the behavior of the components of $\overrightarrow{\mathcal{E}}(t)$ are known, then maximum likelihood methods may be used to find an estimate of $\overrightarrow{\theta_{0}}$. On the other hand, if the variance $V_{0}(t)$ is assumed to vary over time, generalized least squares methods may be used. We assume that $V_{0}=V_{0}(t)$ is constant over time and use an ordinary least squares (OLS) framework as described in [3] to solve the inverse problem. We focus on the vector OLS procedure as we anticipate that our work with the model based on the work in [16] will use multiple observed variables at each time.

Given a mathematical model (1) and discrete statistical model (5) with $n$ time points $t_{j}, j=1,2, \ldots, n, t_{0} \leq t_{1}<$ $t_{2}<\ldots<t_{n} \leq t_{f}$, and $V_{0}=\operatorname{Var}\left(\overrightarrow{\mathcal{E}}\left(t_{j}\right)\right)=\operatorname{diag}\left(\sigma_{0,1}^{2}, \sigma_{0,2}^{2}, \ldots, \sigma_{0, N}^{2}\right)$, we may define

$$
\vec{\theta}_{\mathrm{OLS}}=\underset{\vec{\theta} \in \Theta}{\operatorname{argmin}} \sum_{j=1}^{n}\left[\vec{Y}_{j}-\vec{f}\left(t_{j}, \vec{\theta}\right)\right]^{T} V_{0}^{-1}\left[\vec{Y}_{j}-\vec{f}\left(t_{j}, \vec{\theta}\right)\right],
$$

where $\Theta$ is the set of all possible values of $\vec{\theta}$, to be the OLS minimizer such that each element of the difference vector $\vec{Y}_{j}-\vec{f}\left(t_{j}, \vec{\theta}\right)$ is weighted using the variance of its corresponding observed variable. A realization of this problem using data $\vec{y}_{j}, j=1,2, \ldots, n$, is written

$$
\hat{\theta}_{\mathrm{OLS}}=\underset{\vec{\theta} \in \Theta}{\operatorname{argmin}} \sum_{j=1}^{n}\left[\vec{y}_{j}-\vec{f}\left(t_{j}, \vec{\theta}\right)\right]^{T} V_{0}^{-1}\left[\vec{y}_{j}-\vec{f}\left(t_{j}, \vec{\theta}\right)\right] .
$$

However, calculating $\hat{\theta}_{\text {oLs }}$ still requires the unknown $V_{0}$. If the number of parameters $p$ of a system is sufficiently small and number of observations $n$ large so that $p<n$, then we may calculate the bias adjusted estimate of the variances

$$
V_{0} \approx \hat{V}=\operatorname{diag}\left(\frac{1}{n-p} \sum_{j=1}^{n}\left[\vec{y}_{j}-\vec{f}\left(t_{j}, \hat{\theta}_{\mathrm{OLS}}\right)\right]\left[\vec{y}_{j}-\vec{f}\left(t_{j}, \hat{\theta}_{\mathrm{OLS}}\right)\right]^{T}\right),
$$

and find the estimate of $\vec{\theta}_{0}$ using

$$
\vec{\theta}_{0} \approx \hat{\theta}_{\mathrm{OLS}}=\underset{\vec{\theta} \in \Theta}{\operatorname{argmin}} \sum_{j=1}^{n}\left[\vec{y}_{j}-\vec{f}\left(t_{j}, \vec{\theta}\right)\right]^{T} \hat{V}^{-1}\left[\vec{y}_{j}-\vec{f}\left(t_{j}, \vec{\theta}\right)\right] .
$$


Therefore, finding $\hat{\theta}_{\text {oLS }}$ when $V_{0}$ is unknown requires solving the coupled system of equations (8) and (9).

We may utilize the asymptotic properties of the OLS minimizer (6) to learn about the behavior of the model (1) and (3). As the number of samples $n \rightarrow \infty, \vec{\theta}_{\text {oLs }}$ has the following properties $[11,14]$

$$
\vec{\theta}_{\mathrm{oLS}} \sim \mathcal{N}\left(\vec{\theta}_{0}, \Sigma_{0}^{n}\right) \approx \mathcal{N}\left(\hat{\theta}_{\mathrm{oLS}}, \hat{\Sigma}^{n}\right)
$$

where

$$
\Sigma_{0}^{n} \approx\left(\sum_{j=1}^{n} D_{j}^{T}\left(\vec{\theta}_{0}\right) V_{0}^{-1} D_{j}\left(\vec{\theta}_{0}\right)\right)^{-1}
$$

is the $p \times p$ covariance matrix, and $D_{j}(\vec{\theta})$ is the $N \times p$ matrix

$$
D_{j}(\vec{\theta})=D_{j}^{n}(\vec{\theta})=\left(\begin{array}{cccc}
\frac{\partial f_{1}\left(t_{j}, \vec{\theta}\right)}{\partial \theta_{1}} & \frac{\partial f_{1}\left(t_{j}, \vec{\theta}\right)}{\partial \theta_{2}} & \ldots & \frac{\partial f_{1}\left(t_{j}, \vec{\theta}\right)}{\partial \theta_{p}} \\
\vdots & \vdots & & \vdots \\
\frac{\partial f_{N}\left(t_{j}, \vec{\theta}\right)}{\partial \theta_{1}} & \frac{\partial f_{N}\left(t_{j}, \vec{\theta}\right)}{\partial \theta_{2}} & \ldots & \frac{\partial f_{N}\left(t_{j}, \vec{\theta}\right)}{\partial \theta_{p}}
\end{array}\right) .
$$

The approximation $\hat{\Sigma}^{n}$ to the covariance matrix $\Sigma_{0}^{n}$ is

$$
\Sigma_{0}^{n} \approx \hat{\Sigma}^{n}=\left(\sum_{j=1}^{n} D_{j}^{T}\left(\hat{\theta}_{\mathrm{oLs}}\right) \hat{V}^{-1} D_{j}\left(\hat{\theta}_{\mathrm{oLs}}\right)\right)^{-1} .
$$

We may use $\hat{\Sigma}^{n}$ to approximate the standard errors of each parameter in $\hat{\theta}_{\mathrm{OLS}}$. For the $k$ th element of $\hat{\theta}_{\mathrm{OLS}}$, written $\hat{\theta}_{\mathrm{OLS}, k}$, the standard error is $S E\left(\hat{\theta}_{\mathrm{OLS}, k}\right) \approx \sqrt{\hat{\Sigma}_{k k}^{n}}$, where $\hat{\Sigma}_{k k}^{n}$ is the element in the $k$ th row and $k$ th column of $\hat{\Sigma}^{n}$. Additionally, we define the Fisher Information Matrix to be the inverse of the covariance matrix, which we approximate using (12) by $F\left(\left\{t_{j}\right\}, \vec{\theta}_{0}\right) \approx \hat{F}\left(\left\{t_{j}\right\}, \hat{\theta}_{\mathrm{oLS}}\right)=\left(\hat{\Sigma}^{n}\right)^{-1}$.

\subsection{Choosing optimal state variables to measure}

In many applications, the number of state variables theoretically available to measure may exceed the number of state variables that an experimenter actually has the resources to sample. For example, our dynamical system model for the Calvin Cycle includes 38 distinct metabolites that an investigator could record. However, recording this number of metabolites could be prohibitively expensive. It is therefore desirable to find a subset of the available state variables that is optimal in some sense. We propose two methods for choosing this optimal subset of state variables and explore both of them using simulated data from the photosynthesis problem.

\subsubsection{Simple linear regression}

The first method transforms the perspective of the problem such that we can apply classical variable selection techniques. In our formulation of the mathematical model (1) and statistical model (5), we view the 38 metabolites as the response variables and time and the parameter $\vec{\theta}$ as the covariates (or inputs). If we had data, we would then find an estimate $\vec{\theta}_{O L S}$ of $\vec{\theta}_{0}$ using ordinary least squares and find a model solution that describes the metabolite concentrations over time. There are no known statistical or mathematical methods to select optimal outcome variables, and so in this framework we cannot identify which state variables would be best to measure.

In the Calvin cycle, however, the production of metabolites is not the primary goal of the plant: it is carbon assimilation, which leads to plant growth. Carbon dioxide uptake, which is related to carbon assimilation, may be estimated using a linear combination of the velocity rates of a few of the enzyme reactions. In this conceptual framework, we may now identify which state variables to measure. We define the 38 metabolites to be the covariates and we use the estimated $\mathrm{CO}_{2}$ uptake rate as the response variable. With this change in perspective, we have transformed a complicated problem into a simple problem that may be addressed using standard statistical techniques.

Since the Calvin cycle is a dynamic biological process, it is important to evaluate any model with longitudinal data, i.e., model values at different times. Treating the state variables, metabolite concentrations, as independent variables 
and $\mathrm{CO}_{2}$ uptake as the response, we perform simple linear regression at $n$ selected time points $t_{j}, j=1, \ldots, n$. The regression model for $k \leq N$ predictor variables $M_{i_{1}}, M_{i_{2}}, \ldots, M_{i_{k}}$ is

$$
Y_{j}=\beta_{0}+\beta_{1} M_{i_{1}, j}+\beta_{2} M_{i_{2}, j}+\cdots+\beta_{k} M_{i_{k}, j}+\epsilon_{j}
$$

where $Y_{j}$ is the $\mathrm{CO}_{2}$ uptake rate at time $t_{j}, M_{i_{l}, j}$ is the concentration of metabolite $i_{l}$ at time $t_{j}$, and $N=38$, the number of metabolites.

To determine which metabolites are most strongly associated with the $\mathrm{CO}_{2}$ uptake at a particular time $t_{j}$, variable selection can then be performed using forward, backwards, or stepwise selection using some selection criterion such as the Akaike Information Criterion (AIC), described in [1], or the Bayesian Information Criterion (BIC). The forward selection starts with no predictor variables in the model and sequentially adds the variable with the most explanatory power until all remaining predictors have significance levels below some threshold. The backward selection process starts with all predictor variables included and at each step eliminates the variable with the least explanatory power until all remaining variables have significance above some threshold. Stepwise selection combines the previous two procedures and considers whether to include or exclude the new variable in the model at each step. For each of these methods, a selection criterion (AIC, BIC, etc.) is computed for the model at each step. The final model chosen is the one that optimizes this selection criterion. In this effort, we used the stepwise selection based on the AIC statistic. This statistic provides a means for comparing models with respect to their ability to fit a given data set. It is a relative measure of "information lost" when a given model is used to describe the data. When used with a particular model it gives a quantitative measure of the trade-off between model complexity and model goodness of fit.

AIC [1] is the statistic $A I C=2(k+1)-2 \ln (L)$ where $k$ is the number of predictor variables in the model and $L$ is the value of the likelihood function evaluated at $\theta_{O L S}$. Minimizing AIC over models considered should give a model that both fits the data well and is relatively sparse. Thus, for a given time point, $t_{j}$, we select the model $Y_{j}$ that minimizes AIC and conclude that the predictors in $Y_{j}$ represent the best set of metabolites to measure at $t_{j}$.

A key step required to conduct this optimization procedure is to generate a plausible dataset if multiple sets of experimental data are not available. To obtain multiple records, we choose $n$ time points and for each discrete time point, we generate a dataset of 100 replicates of metabolite concentrations and $\mathrm{CO}_{2}$ uptake rates. This dataset was generated by adding 5\% noise from the standard normal distribution to each metabolite concentration given by the numerically solved model and $\mathrm{CO}_{2}$ uptake rate. We perform this procedure at 10 different times, and then run the variable selection linear regression procedure for each time point. Ultimately, we select the metabolites that appear in the union of each of the 10 models more than three times.

\subsubsection{Optimal design criterion method}

New to the field of experimental design are methods to determine which state variables should be measured before an experiment is performed. Often the observed variables are selected because of the availability and cost of sampling techniques, but in the field of molecular plant biology there are many metabolite assays that may be performed. Here we develop a method that utilizes the Fisher Information Matrix (FIM) that is similar in its framework to the time point selection techniques used in optimal design of experiments in [8].

Recall that in (7) we search for the parameter vector $\hat{\theta}_{\text {oLs }}$ that minimizes

$$
J_{d}(\vec{y}, \vec{\theta})=\sum_{j=1}^{n}\left[\vec{y}_{j}-\vec{f}\left(t_{j}, \vec{\theta}\right)\right]^{T} V_{0}^{-1}\left[\vec{y}_{j}-\vec{f}\left(t_{j}, \vec{\theta}\right)\right]
$$

the OLS cost function weighted by the variances $V_{0}$ of the error processes $\overrightarrow{\mathcal{E}}$. Emphasizing the presence of the observation operator $\mathbf{L}=\mathcal{C}$, where the projection $\mathbf{L}: \mathbb{R}^{m} \rightarrow \mathbb{R}^{N}$ is a linear transformation, we write (14) as

$$
J_{d}(\vec{y}, \vec{\theta}, \mathbf{L})=\sum_{j=1}^{n}\left[\mathbf{L}\left(\vec{x}\left(t_{j}, \vec{\theta}_{0}\right)+\vec{\epsilon}_{j}\right)-\mathbf{L} \vec{x}\left(t_{j}, \vec{\theta}\right)\right]^{T} V_{0}^{-1}\left[\mathbf{L}\left(\vec{x}\left(t_{j}, \vec{\theta}_{0}\right)+\vec{\epsilon}_{j}\right)-\mathbf{L} \vec{x}\left(t_{j}, \vec{\theta}\right)\right] .
$$

We construct $\mathbf{L}$ using row vectors $\vec{l}_{\alpha}, 1 \leq \alpha<N$, where element $\beta$ in $\vec{l}_{\alpha}$, denoted $l_{\alpha}(\beta)$, describes the weight of state variable $\beta$ in observation variable $\alpha$. Choose $N$ such observation method vectors. Then $\mathbf{L}$ is a $N \times m$ matrix such that $\mathbf{L}_{\alpha, \beta}=0$ if state variable $\beta$ is not measured in observed variable $\alpha$ and $\mathbf{L}_{\alpha, \beta}=\psi$ if state variable $\beta$, $1 \leq \beta \leq m$, is measured in observed variable $\alpha, 1 \leq \alpha \leq N$, with a weight of $\psi$ that is determined by the observation 
method. In practice, $\mathbf{L}$ would be sparse and include only one nonzero element in a row or a few nonzero elements per row, representing in this latter case that a linear combination of state variables are measured in a particular observation operator. Additionally, as it does not benefit the experimenter to perform the same measurement twice at one time point, it is likely that $\mathbf{L}$ has full row rank. In order to compare the effect of $\mathbf{L}$ on our ability to accurately estimate $\vec{\theta}_{0}$, we write the FIM as

$$
F\left(\vec{\theta}_{0}, \mathbf{L}\right)=\sum_{j=1}^{n}\left(\mathbf{L} D_{j}\left(\vec{\theta}_{0}\right)\right)^{T} V_{0}^{-1}\left(\mathbf{L} D_{j}\left(\vec{\theta}_{0}\right)\right)
$$

where

$$
D_{j}(\vec{\theta})=D_{j}^{n}(\vec{\theta})=\left(\begin{array}{cccc}
\frac{\partial x_{1}\left(t_{j}, \vec{\theta}\right)}{\partial \theta_{1}} & \frac{\partial x_{1}\left(t_{j}, \vec{\theta}\right)}{\partial \theta_{2}} & \ldots & \frac{\partial x_{1}\left(t_{j}, \vec{\theta}\right)}{\partial \theta_{p}} \\
\vdots & \vdots & & \vdots \\
\frac{\partial x_{m}\left(t_{j}, \vec{\theta}\right)}{\partial \theta_{1}} & \frac{\partial x_{m}\left(t_{j}, \vec{\theta}\right)}{\partial \theta_{2}} & \ldots & \frac{\partial x_{m}\left(t_{j}, \vec{\theta}\right)}{\partial \theta_{p}}
\end{array}\right) .
$$

We select an $N<m$, and let $\mathcal{L}_{N}$ be the set of all matrices $\mathbf{L}$ as defined above. Assume we may formulate a functional $\mathcal{J}^{N}: \mathbb{R}^{p \times p} \rightarrow \mathbb{R}^{+}$. Then the optimal design problem associated with the functional $\mathcal{J}^{N}$ is selecting a matrix $\hat{\mathbf{L}}$ such that

$$
\mathcal{J}^{N}\left(F\left(\vec{\theta}_{0}, \hat{\mathbf{L}}\right)\right)=\min _{\mathbf{L} \in \mathcal{L}_{N}} \mathcal{J}^{N}\left(F\left(\vec{\theta}_{0}, \mathbf{L}\right)\right)
$$

We observe that $\mathcal{L}_{N}$ is a finite set if the weights $\psi$ are given and fixed. (Although we don't do so here, one could, of course, also optimize over a weighting set $\Psi=\{\psi\}$ and this could, in general cases, lead to an infinite dimensional optimization problem similar to those formulated in [7].) In any event, solving the corresponding optimal design problem is conceptually simple. Using (16), we create the set $\mathcal{F} \subset \mathbb{R}^{p \times p}$ of all possible FIM $F$ that could be formulated from the observation matrices $\mathbf{L} \in \mathcal{L}_{N}$. By the properties of matrix multiplication and addition, this set is also finite. Then the functional $\mathcal{J}^{N}$ applied to all $F \in \mathcal{F}$ produces a finite set contained in $\mathbb{R}^{+}$. Because this set is finite, it is well-ordered by the relation $\leq$ and therefore has a minimal element. Therefore we may find at least one solution, $\hat{\mathbf{L}}$, to $(17)$.

We may consider the use of several different functionals in the context of D-optimal, E-optimal, c-optimal, or SEoptimal design criteria and also the matrix norm [7] to determine the form of $\mathcal{J}^{N}$. Many of these design criteria use the inverse of the FIM, which is equivalently the approximate covariance matrix.

In D-optimal design,

$$
\mathcal{J}_{D}^{N}(F) \equiv \operatorname{det}\left(F\left(\vec{\theta}_{0}, \mathbf{L}\right)^{-1}\right)=\frac{1}{\operatorname{det}\left(F\left(\vec{\theta}_{0}, \mathbf{L}\right)\right)}
$$

By minimizing $\mathcal{J}_{D}^{N}$, we minimize the volume of the confidence interval ellipsoid describing the uncertainty in our parameter estimates. Since $F$ is symmetric and positive semi-definite, $\mathcal{J}_{D}^{N}(F) \geq 0$. Additionally, since $F$ is invertible, $\mathcal{J}_{D}^{N}(F) \neq 0$, therefore, $\mathcal{J}_{D}^{N}: \mathbb{R}^{p \times p} \rightarrow \mathbb{R}^{+}$.

In E-optimal design, the cost functional is $\mathcal{J}_{E}^{N}$ is the largest eigenvalue of $\left(F\left(\vec{\theta}_{0}, \mathbf{L}\right)\right)^{-1}$, or equivalently,

$$
\mathcal{J}_{E}^{N}(F)=\max \frac{1}{\operatorname{eig}\left(F\left(\vec{\theta}_{0}, \mathbf{L}\right)\right)} .
$$

To obtain a smaller $\mathcal{J}_{E}^{N}$, we must reduce the length of the principal axis of the confidence interval ellipsoid. Since an eigenvalue $\lambda$ solves $\operatorname{det}(F-\lambda I)=0$, an eigenvalue of $\lambda=0$ would mean $\operatorname{det}(F)=0$, or that $F$ is not invertible. Since $F$ is positive semi-definite, all eigenvalues are therefore positive. Thus also $\mathcal{J}_{E}^{N}: \mathbb{R}^{p \times p} \rightarrow \mathbb{R}^{+}$.

In c-optimal design, the cost functional is written $\mathcal{J}_{c}^{N}(F)=c^{T}\left(F\left(\vec{\theta}_{0}, \mathbf{L}\right)\right)^{-1} c$, where $c \in \mathbb{R}^{p}$ describes the importance of accurately estimating the parameters relative to the other parameters. Minimizing $\mathcal{J}_{c}^{N}$ is equivalent to minimizing the variances of the parameters with the largest elements in $c$. Since $F$ and $F^{-1}$ are positive definite, the quadratic form is positive, convex, and has a global minimum. Therefore $\mathcal{J}_{c}^{N}: \mathbb{R}^{p \times p} \rightarrow \mathbb{R}^{+}$.

In SE-optimal $[7,8]$ design $\mathcal{J}_{S E}^{N}$ is a weighted sum of the elements on the diagonal of $\left(F\left(\vec{\theta}_{0}, \mathbf{L}\right)\right)^{-1}$, written

$$
\mathcal{J}_{S E}^{N}(F)=\sum_{i=1}^{p} \frac{\left(F\left(\vec{\theta}_{0}, \mathbf{L}\right)\right)_{i, i}^{-1}}{\theta_{0, i}^{2}} .
$$


Thus in SE-optimal design, the goal is to minimize the sum of squared errors of the parameters normalized by the true parameter values. As the diagonal elements of $F^{-1}$ are all non-negative, we also have $\mathcal{J}_{S E}^{N}: \mathbb{R}^{p \times p} \rightarrow \mathbb{R}^{+}$.

When using a norm to describe the cost function, the squared matrix norm $\|\cdot\|_{2}^{2}$ is typically used. However, minimizing $\left\|F^{-1}\right\|_{2}^{2}=\max \left\{\operatorname{eig}\left(\left(F^{-1}\right)^{2}\right)\right\}$ will give the same results as E-optimal design.

We believe that one could also use currently undeveloped techniques related to sensitivity analysis to determine which state variables are most strongly associated with the parameters. In the next section, we employ mathematical and statistical techniques to first determine which parameters have the largest impact on the behavior of the dynamical system. Then, we determine which state variables are most important to measure. The first method, which utilizes linear regression and the Akaike Information Criterion [1], identifies which state variables are most strongly associated with the outcome of interest, carbon dioxide assimilation. The second, which minimizes an optimal design criterion based on the Fisher Information Matrix, identifies which state variables provide sufficient information to accurately estimate a subset of parameters.

\section{Parameter Selection and Estimation}

\subsection{Sensitivity analysis results}

Broadly defined, sensitivity analysis is a set of techniques that can be used to assess the dependence of a model's outputs on parameter values. In biological problems, the number of parameters are often sufficiently high to where it is computationally costly or even impossible to attempt to estimate all of them in the inverse problem. In such situations, it is necessary to choose some parameters to estimate and fix the remaining parameters at reasonable estimates. Sensitivity analysis can be employed to help make such decisions. The $m \times p$ sensitivity matrix, $D_{j}(\vec{\theta})$, contains the values of the sensitivity functions evaluated at time $t_{j}$ and is defined in (11). The sensitivity matrix describes how much each component of the vector $\vec{f}$ is dependent upon a particular component of the parameter vector $\vec{\theta}$ at a particular time $t_{j}$. Sensitivity matrices for large systems are typically calculated using finite difference techniques or automatic differentiation.

Plotting the values of these matrices using a heat map can give us substantial information with minimal additional effort. When an element $\frac{\partial f_{i}\left(t_{j}, \vec{\theta}\right)}{\partial \theta_{k}}$ in the sensitivity matrix is far away from 0 , it indicates that the value of parameter $k$ has a substantial impact on the output of function $f_{i}$ at time $t_{j}$. Thus, we can identify which parameters we should estimate as well as which time intervals the parameters have the largest impact on the dynamical system. Several additional algorithms for choosing the most important parameters are discussed in [2] and [10]. These methods choose a subset of parameters that minimize a selection score related to the covariance matrix of the system while maintaining a full rank sensitivity matrix corresponding to the subset of included parameters. The proposed algorithms rely on searching each potential set of parameters, however, and are therefore computationally costly, requiring up to

$$
\sum_{p=1}^{p_{0}}\left(\begin{array}{c}
p_{0} \\
p
\end{array}\right)
$$

comparisons, where $p_{0}$ is the number of parameters in the model of interest. Additionally, [2] provides theory for only scalar data, not data with multiple observed variables.

We chose to implement the simpler graphing method to determine which parameters were most important in our plant model. Using finite differences, we evaluate the sensitivity functions at a discrete set of time points using the parameter values given in [16] to create the sensitivity matrices $D_{j}(\vec{\theta})$, calculate the sum $\sum_{j=1}^{n}\left|D_{j}(\vec{\theta})\right|$, and identify parameters that are associated with the largest elements in $\sum_{j=1}^{n}\left|D_{j}(\vec{\theta})\right|$. This set of parameters most strongly influences the behavior of the dynamical system over time.

We first generated sensitivity matrices $D_{j}$ for five different sets of ten discrete time points $t_{j}$. For each set of time points, we then summed the absolute value of these ten matrices to select the parameters for which the system was most sensitive. We discovered that KM11, KM521, KI523, KC, KM1221 and KM1241 elicited the largest sensitivity values for all five sets of ten time points. KM12, KM13, KE4, KM131, KE22, KM521 and V58 appeared to generate nontrivial sensitives in some (but not all) of the sets of ten time points. We use the results of this analysis in the next section of parameter analysis. 


\subsection{Parameter estimation}

Before we may conclusively identify the optimal times at which to sample or the optimal metabolites to sample, we must first test the feasibility of predicting a small number of parameters in the Zhu Calvin Cycle model [16]. Since we do not have a set of data, we use the simulated solution to this model, with parameter vector $\theta_{0}$ with values as described in [16], to calculate eleven data points of all 38 metabolites at times $t_{j}=\frac{j-1}{10} 5000, j=1,2, \ldots, 11$, so that $T_{f}=5000$ seconds. To perform the inverse problem, we add or subtract $25 \%$ of the true value of the parameters we will estimate and then use unconstrained nonlinear optimization via a simplex search to minimize the unweighted least squares error between the predicted solution and the true solution that uses $\theta_{0}$.

We perform the inverse problem for the parameter subsets

$$
\begin{aligned}
\theta_{0 a}= & {[K M 11, K M 521, K I 523, K C, K M 1221, K M 1241], } \\
\theta_{0 b}= & {[K M 11, K M 12, K M 13, K E 4, K M 131, K E 22, K M 511, K M 521,} \\
& K I 523, K C, K M 1221, K M 1241, V 58], \\
\theta_{0 c}= & {\left[R u B P_{0}, S B P_{0}, K M 11, K M 12, K M 13, K I 13, K E 4, K M 9, K M 131, K I 135, K E 22,\right.} \\
& K M 511, K M 514, K M 521, K I 523, K C, K M 1221, K M 1241, V 9, V 58, V 111],
\end{aligned}
$$

and record (in Table 1) the parameter estimates $\hat{\theta}_{a}^{ \pm}, \hat{\theta}_{b}^{ \pm}$, and $\hat{\theta}_{c}^{ \pm}$, respectively, for the initial parameter guesses at $1 \pm .25$ times the parameter subset true values. We also record the least squares error and the runtime for the inverse problem as performed on a laptop with a $2.67 \mathrm{GHz}$ 4-core Intel i7 processor and $4 \mathrm{~GB}$ RAM. We plot the $\mathrm{CO}_{2}$ uptake rates for the true solution and the solution generated by the parameter estimates in Figures 1-3.

Estimation of six parameters to which the model is most sensitive provided mixed results when only $25 \%$ away from the true value. Starting at $25 \%$ below $\theta_{0}$, the minimization routine was able to estimate KM11, KI523, and KC very well; however, starting at $25 \%$ above $\theta_{0}$ led to an estimate $\hat{\theta}_{a}^{+}$that is not near $\theta_{0}$ (Table 1 ). The variability in results may be due to the capabilities of fminsearch or undetected interactions between parameters. For example, when estimating KM521, perhaps KI521 should also be estimated. When estimating the top 13, all parameters could be estimated reasonably well with the exception of KM12, which was far from its true value in both $\hat{\theta}_{b}^{-}$and $\hat{\theta}_{b}^{+}$, KM521, $\mathrm{KC}$, and $\mathrm{KM} 1221$. The $\mathrm{CO}_{2}$ uptake rates of the models generated by $\hat{\theta}_{b}^{-}$and $\hat{\theta}_{b}^{+}$, however, appear to be very close to that of the model generated by $\theta_{0}$ (Figure 2). Adding parameter constraints would aid in producing biologically correct parameter estimates. Estimation of 21 parameters (the top 13 plus 8 more parameters used in describing reactions that involve RuBP and SBP, which are seen to be related to Rubisco in [16]) was surprisingly effective, with only 6 values in $\hat{\theta}_{c}^{-}$and 2 in $\hat{\theta}_{c}^{+}$being far from their true values (Table 1). The performance of the model generated by $\hat{\theta}_{c}^{+}$is also impressive (Figure 3). Since some of these poorly estimated parameters are also problematic in the 13-parameter case, we may want to refocus our efforts on other parameters or perform more refined sensitivity analysis.

It appears that if the runtime of the parameter estimation problem is a concern, it would be best to estimate no more than 10 parameters; however, the results are sometimes far from the true parameters. Having correct parameter estimates in this context is especially important, as different reaction rates would paint a different picture of how the plant is performing. It is possible to estimate a large number of parameters (over 20) if reasonable initial guesses can be provided. Additionally, the number of parameters that could be estimated - and the quality of those estimates - could be improved if constraints were imposed.

\section{Optimal metabolites to measure}

Focusing on the overall $\mathrm{CO}_{2}$ uptake rate of the plant and on the parameters identified through sensitivity analysis as most important to the differential equation system, we perform our two metabolite identification techniques: simple linear regression to create a linear model of how metabolites relate to $\mathrm{CO}_{2}$ uptake at selected time points, and optimal design criterion minimization of a functional $\mathcal{J}^{N}$ on the Fisher Information Matrix.

\subsection{Simple linear regression}

Using the simple linear regression (SLR) variable selection technique; we obtain the set of metabolites that best predict the $\mathrm{CO}_{2}$ update rate at each of 10 evenly spaced time points $t_{j}$ between 0 and 3000 seconds. We considered the metabolites that appeared often ( 3 times or more), over all of the models for each time point, as the most important 


\begin{tabular}{|r|c|cc|cc|cc|}
\hline Name & $\theta_{0}$ value & $\hat{\theta}_{a}^{-}$ & $\hat{\theta}_{a}^{+}$ & $\hat{\theta}_{b}^{-}$ & $\hat{\theta}_{b}^{+}$ & $\hat{\theta}_{c}^{-}$ & $\hat{\theta}_{c}^{+}$ \\
\hline \hline KM11 & 0.0115 & 0.0115 & 0.0108 & 0.009 & 0.0139 & 0.0081 & 0.0140 \\
\hline KM12 & 0.222 & - & - & 0.1447 & 0.4354 & 0.1275 & 0.377 \\
\hline KM13 & 0.02 & - & - & 0.198 & 0.0298 & 0.0170 & 0.0276 \\
\hline KE4 & 0.05 & - & - & 0.0502 & 0.05 & 0.049 & 0.0502 \\
\hline KM131 & 0.05 & - & - & 0.0492 & 0.0499 & 0.0354 & 0.0561 \\
\hline KE22 & 0.058 & - & - & 0.0593 & 0.0577 & 0.0547 & 0.0581 \\
\hline KM511 & 0.02 & - & - & 0.0194 & 0.02 & 0.0203 & 0.0201 \\
\hline KM521 & 0.0025 & 0.0201 & 0.0097 & $5.5 \mathrm{e}-4$ & 0.0022 & $4 \mathrm{e}-5$ & 0.0031 \\
\hline KI523 & $7 \mathrm{e}-5$ & $7.1 \mathrm{e}-5$ & $2.2 \mathrm{e}-5$ & $2.7 \mathrm{e}-5$ & $6.2 \mathrm{e}-5$ & $1 \mathrm{e}-6$ & $8.5 \mathrm{e}-5$ \\
\hline KC & 0.0115 & 0.0114 & 0.0425 & 0.0114 & 0.0116 & 0.0062 & 0.0021 \\
\hline KM1221 & 0.15 & 0.0813 & 0.0023 & 0.1247 & 0.2096 & 0.1007 & 0.1894 \\
\hline KM1241 & 0.15 & 0.0894 & 0.0236 & 0.1276 & 0.2027 & 0.1064 & 0.1848 \\
\hline V58 & 0.0168 & - & - & 0.0098 & 0.0168 & 0.0192 & 0.0174 \\
\hline \hline Runtime(sec) & & 282.09 & 362.80 & 854.20 & 640.53 & 1940.63 & 2115.75 \\
\hline LSQ Error & & 0.1429 & 88.98 & 0.9895 & 0.02649 & 0.1700 & 0.04131 \\
\hline
\end{tabular}

Table 1: True values $\theta_{0}$ and parameter estimates for the top 13 parameters
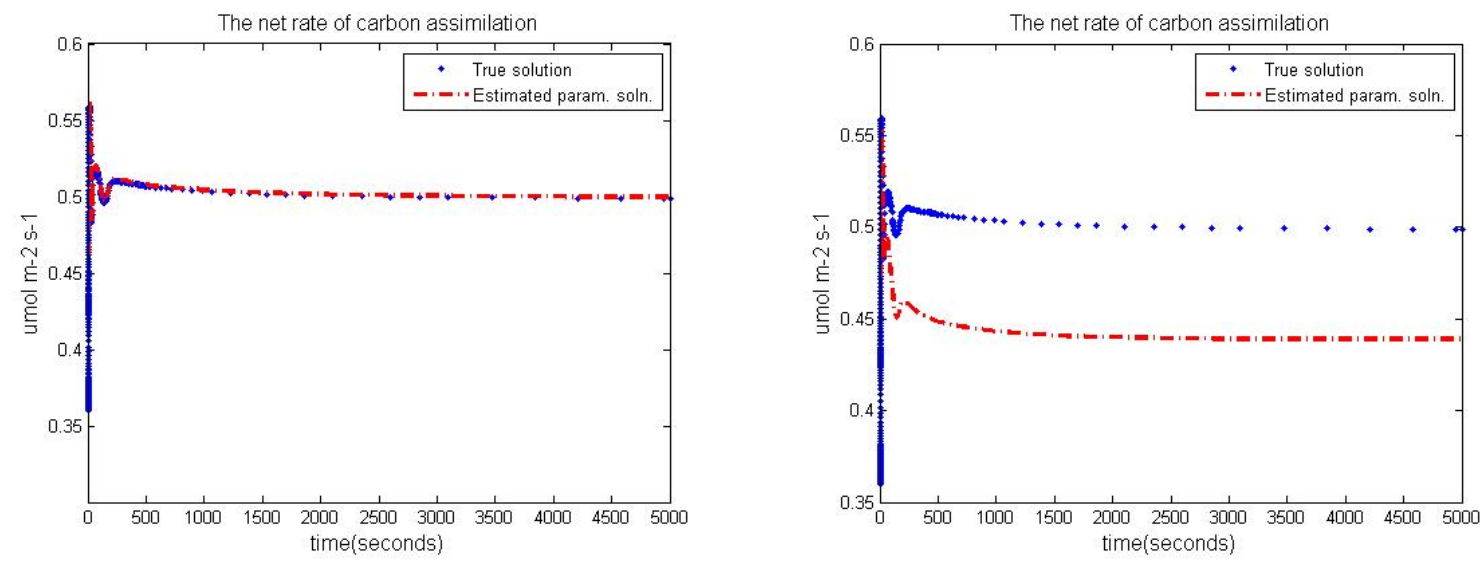

Figure 1: $\mathrm{CO}_{2}$ uptake rates for $\theta_{0}$ (blue dots) and estimates $\hat{\theta}_{a}^{-}$(left, red dash) and $\hat{\theta}_{a}^{+}$(right, red dash).

features. These metabolites are underlined in Table 2: RuBP, NADPH, GCEA, F26BPc, T3P, Goac, SUC, FBP, CO2, PGCA, GLUc, NADHc, T3Pc. Since these metabolites most closely associated with $\mathrm{CO}_{2}$ uptake rate, we suggest measurement of these metabolites for an optimal fit. 

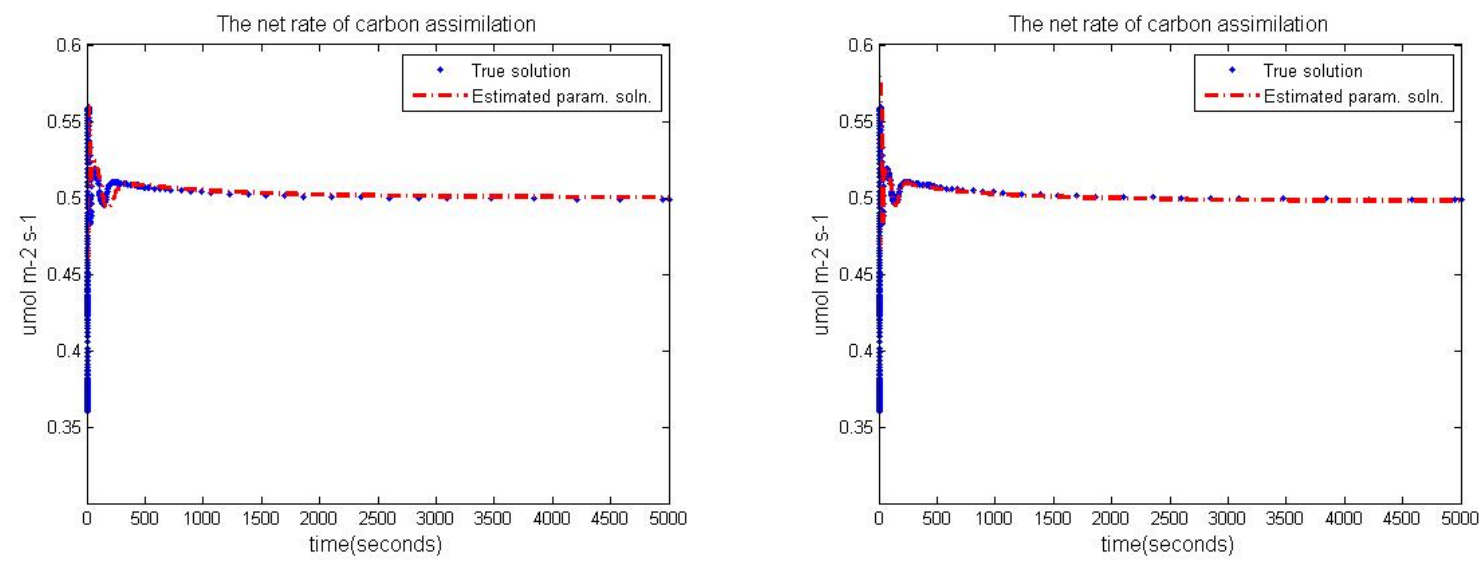

Figure 2: $\mathrm{CO}_{2}$ uptake rates for $\theta_{0}$ (blue dots) and estimates $\hat{\theta}_{b}^{-}$(left, red dash) and $\hat{\theta}_{b}^{+}$(right, red dash).
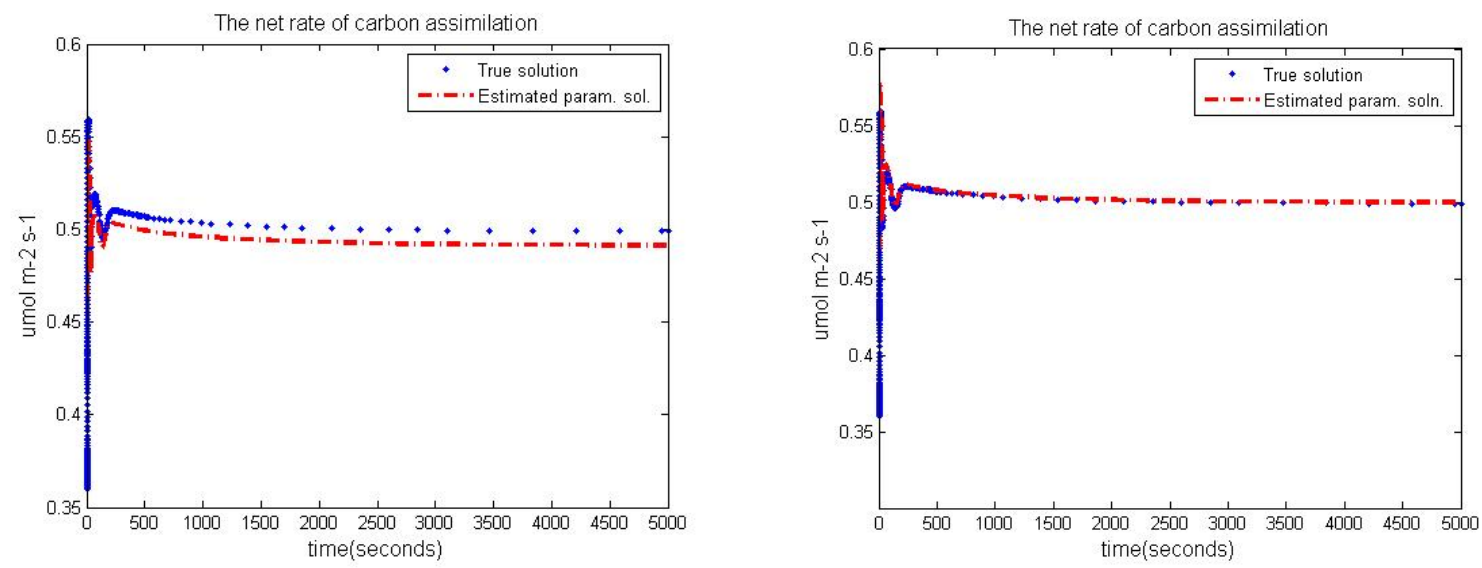

Figure 3: $\mathrm{CO}_{2}$ uptake rates for $\theta_{0}$ (blue dots) and estimates $\hat{\theta}_{c}^{-}$(left, red dash) and $\hat{\theta}_{c}^{+}$(right, red dash).

\begin{tabular}{|c|c|}
\hline Model & Metabolites \\
\hline$T_{1}(\vec{x})$ & RuBP, E4P, ATP, NADPH, GCEA, GCEAc, HexPc, F26BPc, UTPc \\
\hline$T_{2}(\vec{x})$ & 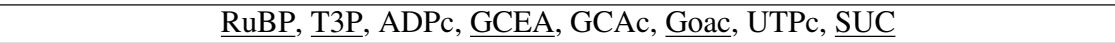 \\
\hline$T_{3}(\vec{x})$ & $\underline{\mathrm{T} 3 \mathrm{P}}, \underline{\mathrm{FBP}}, \underline{\mathrm{CO} 2}, \mathrm{GCA}, \underline{\mathrm{PGCA}}, \underline{\mathrm{Goac}}, \mathrm{HPRc}, \underline{\mathrm{F} 26 \mathrm{BPc}}, \underline{\mathrm{SUC}}$ \\
\hline$T_{4}(\vec{x})$ & NADPH, GLUc, GCEA, PGCA, GLYc, HexPc, SUCP, SUC \\
\hline$T_{5}(\vec{x})$ & T3P, $\underline{\text { CO2}}$, HexP, NADHc, PGCA, T3Pc \\
\hline$T_{6}(\vec{x})$ & RuBP, CO2, NADHc, GCAc, SERc, HPRc, T3Pc, F26BPc, SUC \\
\hline$T_{7}(\vec{x})$ & SBP, NADc, GLUc, GCEA, GCA, SERc, UDPGc \\
\hline$T_{8}(\vec{x})$ & $\underline{\mathrm{RuBP}}, \underline{\mathrm{FBP}}, \underline{\mathrm{NADPH}}, \underline{\mathrm{CO}}, \mathrm{NADc}, \mathrm{ADPc}, \underline{\mathrm{GLUc}}, \underline{\mathrm{GCEA}}, \underline{\mathrm{Goac}}, \mathrm{GLYc}, \mathrm{FBPc}, \mathrm{UDPGc}$ \\
\hline$T_{9}(\vec{x})$ & O2, GCAc, HPRc, GCEAc, HexPc \\
\hline$T_{1} 0(\vec{x})$ & 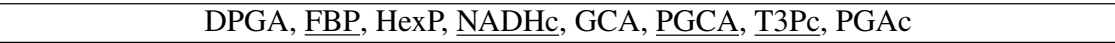 \\
\hline
\end{tabular}

Table 2: The resultant covariates selected by the step-wise selection algorithm for each model at each of the 10 time points. The underlined metabolites represent those that appear multiple (three times or more) times over the 10 models. These highlighted metabolites produce the best fits and therefore represent optimal metabolites to measure. 


\subsection{Optimal design criterion method}

Unlike the simple linear regression method, utilizing the optimal design criterion to identify optimal state variables does not require data; therefore we do not create simulated data. We use the parameter values as described in [16] and allow only the parameters in the parameter sets $\theta_{a}=[\mathrm{KM} 11, \mathrm{KM} 521, \mathrm{KI} 523, \mathrm{KC}, \mathrm{KM} 1221, \mathrm{KM} 1241]$ or $\theta_{c}=\left[\mathrm{RuBP}_{0}, \mathrm{SBP}_{0}\right.$, KM11, KM13, KI13, KE4, KM9, KM131, KI135, KE22, KM511, KM521, KI523, KC, KM1221, KM1241, V9, V58] to vary. We fix the data collection time points at $t_{j}=\frac{j-1}{10} 3000, j=1,2, \ldots, 11$, so that $t_{0}=0$ and $t_{f}=3000$ seconds when using $\theta_{a}$, and time points $t_{j}=\frac{j-1}{10} 3000, j=1,2, \ldots, 21$ for $\theta_{c}$, and consider subsets of $N=3,5,10$ metabolites for both $\theta_{a}$ and $\theta_{c}$ and also $N=15$ for $\theta_{a}$. Each metabolite is assigned a variance of $5 \%$ of its initial value, and to reduce computation time, all metabolites that do not change in concentration over time $(d x / d t=0)$ are excluded from the search algorithm.

According to the estimated standard errors, the D-optimal design criterion performs the best on the Zhu Calvin Cycle model. Solutions generated by D-optimal design tends to minimize the largest standard error (far right columns of Tables 3 and 4), and at times results in a standard error that is at least an order of magnitude better than the corresponding Eoptimal and SE-optimal design solutions.

When estimating a small set of parameters, $\theta_{a}$, only a small number of variables must be measured. Sampling more variables does not decrease standard errors by a great amount - sometimes it even increases the standard errors of some parameters. If only $N=3$ variables were sampled, all three optimal design criteria selected the metabolites PGA, Serc, and F26BPc. When sampling only $N=5$ variables, all three optimization criteria identified PGA, Serc, F26BPc, and $\mathrm{T} 3 \mathrm{P}$ or T3Pc as important variables to measure, and two out of three criteria identified FBPc. This indicates that even when using different optimization criteria, some variables are generally important to measure. This amount of overlap increases as the $N$ increases. In Table 3, one parameter, $\mathrm{KC}$ (the Michaelis constant for $\mathrm{CO}_{2}$ ), consistently has the lowest variance. In our calculations, $\mathrm{KC}=0.0115$, so a variance below the order of $1 \mathrm{e}-3$ is desirable. Similarly, a second parameter, KM521 (a Michaelis constant related to FBP), consistently has the highest standard error. The value used in our calculations is KM521 $=0.0025$. Since the calculated standard errors are so large compared to the parameter value, it would be wise to fix that parameter and instead estimate another parameter. The standard error of KM521 is lower when FBPc or F26BPc are selected to be measured, so if KM521 must be estimated, measuring these two metabolites will theoretically improve the accuracy of the parameter estimate.

The trends seen in the standard errors for the parameters $\theta_{a}$ reflect the results of Section 4.2. The parameters KC and KM11 (which had a standard error almost as low as that of KC) were estimated close to their true values for $\theta_{0 a}$, and KM521 was not well estimated. This indicates that the model is not very sensitive to KM521 at the chosen time points. The calculated standard errors of KM1221 and 1241 were larger than the true parameter value of 0.15 , also matching the somewhat poor estimation of these parameters in the inverse problem.

When estimating a large set of parameters, $\theta_{c}$, we see that we will need to sample a larger number of metabolites (Table 4). At $N=3$, all three selection criterion chose RuBP, SBP, and F26BPc. This indicates that measuring those three variables is critical to estimating the parameters in $\theta_{c}$, but the maximum standard error is very high. This trial used $K I 135=0.4$, and so a standard error with a positive order of magnitude is not desirable. Many other standard errors also had positive orders of magnitude while the initial parameters were between 0 and 1 . Similarly, at $N=5$, RuPB, SBP, GLYc, T3Pc, and F26BPc are selected by multiple optimization criteria, showing that some state variables are generally important to measure in order to have small standard errors. While at $N=5$ the maximum standard error was large, many standard errors decreased by at least an order of magnitude. Most standard errors have an order of magnitude less than 0 if $N=10$ variables are chosen. The overlap in variable selections continue: all three criteria choose RuBP, T3P, SBP, GCEA, SERc, and F26BPc, and many variables chosen by one selection criteria are also chosen by a second. To further reduce standard errors, it may be wise to remove KI135 from the parameter set, as it consistently has the highest standard error. Alternatively, more time points may be sampled, or more variables may be measured.

The selection of $N=10$ state variables for $\theta_{c}$ overlaps with the 12 metabolites frequently selected by the Akaike Information Criterion (AIC) used in the simple linear regression (SLR) technique in Section 5.1: RuBP, T3P, GCEA, and F26BPc are chosen by all three optimal design criteria and by the AIC. Additionally, Goac and T3Pc are selected by two optimal design criteria and the AIC. Additionally, the standard errors calculated in this method reflect the variability in parameter estimates generated in Section 4.2. Parameters that often were estimated far from the true value in Section 4.2 often had higher standard errors, and those that were accurately estimated had smaller standard errors. 


\begin{tabular}{|c|c|c|c|c|c|}
\hline $\mathbf{N}$ & Criterion & Cost & Observables & $\min (\mathrm{SE})$ & $\max (\mathbf{S E})$ \\
\hline 3 & $\mathrm{D}$ & $1.3 \mathrm{e}-26$ & PGA, Serc, F26BPc & KC:0.0379 & KM521:4.182 \\
\hline 5 & $\mathrm{D}$ & $1.8 \mathrm{e}-30$ & PGA,T3P,Goac,Serc, F26BPc & KC:0.0012 & KM521:4.176 \\
\hline 10 & $\mathrm{D}$ & $6.8 \mathrm{e}-31$ & $\begin{array}{l}\text { RuBP, PGA, T3P, E4P, SBP, Goac, } \\
\text { Serc, Glyc, T3Pc, F26BPc }\end{array}$ & KC:0.00089 & KM521:4.038 \\
\hline 15 & $\mathrm{D}$ & $6.17 \mathrm{e}-31$ & $\begin{array}{l}\text { RuBP, PGA, T3P, E4P, S7P, } \\
\text { SBP, PenP, GCEA, Goac, Serc, } \\
\text { Glyc, GCEAc, T3Pc, F26BPc }\end{array}$ & KC:0.00089 & KM521:3.966 \\
\hline 3 & $\mathrm{E}$ & 17.51 & PGA, Serc, F26BPc & KC:0.0379 & KM521:4.182 \\
\hline 5 & $\mathrm{E}$ & 16.05 & PGA, Serc, T3Pc, FBPc, F26BPc & KC:0.0353 & KM521:4.004 \\
\hline 10 & $\mathrm{E}$ & 1.437 & $\begin{array}{c}\text { DPGA, T3P, S7P, ATP, NADPH, } \\
\text { NADHc, GCEA, GCA, Goac, HexPc }\end{array}$ & KC:0.0106 & KM521:53.30 \\
\hline 15 & $\mathrm{E}$ & 2.013 & $\begin{array}{c}\text { DPGA, T3P, FBP, E4P, S7P, } \\
\text { ATP, HexP, GCEA, GCA, PGCA, } \\
\text { GCAc, Goac, HPRc, GCEAc, UDPGc }\end{array}$ & $\mathrm{KC}: 0.004$ & KM521:40.77 \\
\hline 3 & SE & 5608706 & PGA, Serc, F26BPc & KC:0.0379 & KM521:4.182 \\
\hline 5 & SE & 5139737 & PGA, Serc, T3Pc, FBPc, F26BPc & KC:0.0353 & KM521:4.004 \\
\hline 10 & SE & 11543 & $\begin{array}{l}\text { DPGA, FBP, E4P, S7P, ATP, } \\
\text { HexP, GCA, Goac, Serc, T3Pc }\end{array}$ & KC:0.0033 & KM521:16.63 \\
\hline 15 & SE & 292234 & $\begin{array}{c}\text { DPGA, T3P, FBP, E4P, S7P, } \\
\text { ATP, HexP, GCEA, GCA, PGCA } \\
\text { GCAc, Goac, HPRc, GCEAc, UDPGc }\end{array}$ & KC:0.004 & KM521:40.77 \\
\hline
\end{tabular}

Table 3: Number of observables, optimal design criterion, optimal cost, selected observables, and the minimum and maximum standard error and associated parameter for the parameter subset $\theta_{a}$.

\begin{tabular}{|c|c|c|c|c|c|}
\hline $\mathbf{N}$ & Criterion & Cost & Observables & $\min (\mathrm{SE})$ & $\max (\mathrm{SE})$ \\
\hline 3 & $\mathrm{D}$ & $1.2 \mathrm{e}-11$ & RuBP, SBP, F26BPc & $\mathrm{SBP}_{0}: 0.1225$ & KI135:2642 \\
\hline 5 & $\mathrm{D}$ & $1.5 \mathrm{e}-44$ & RuBP, T3P, SBP, SERc, F26BPc & V58:0.0883 & KI135:287.1 \\
\hline 10 & $\mathrm{D}$ & $5.8 \mathrm{e}-68$ & $\begin{array}{c}\text { RuBP, T3P, SBP, PenP, GCEA, } \\
\text { Goac, SERc, GLYc, T3Pc, F26PBc }\end{array}$ & KM511:0.0041 & KI135:19.10 \\
\hline 3 & $\mathrm{E}$ & $7.1 \mathrm{e} 6$ & RuBP, SBP, F26BPc & $\mathrm{SBP}_{0}: 0.1225$ & KI135:2642 \\
\hline 5 & $\mathrm{E}$ & 2092 & RuBP, SBP, PenP, GLYc, T3Pc & $\mathrm{SBP}_{0}: 0.1225$ & KI135:40.93 \\
\hline 10 & $\mathrm{E}$ & 287.8 & $\begin{array}{c}\text { RuBP, PGA, T3P, S7P, SBP, } \\
\text { PenP, GCEA, SERc, GCEAc, F26BPc }\end{array}$ & V58:0.0097 & KI135:16.91 \\
\hline 3 & SE & $1.3 \mathrm{e} 9$ & RuBP, SBP, F26BPc & $\mathrm{SBP}_{0}: 0.1225$ & KI135:2642 \\
\hline 5 & SE & $4.2 \mathrm{e} 6$ & RuBP, SBP, GLYc, T3Pc, F26BPc & KM511:0.0446 & KI135:548.8 \\
\hline 10 & $\mathrm{SE}$ & $3.8 \mathrm{e} 11$ & $\begin{array}{c}\text { RuBP, PGA, T3P, SBP, GCEA, } \\
\text { Goac, SERc, T3Pc, FBPc, F26BPc }\end{array}$ & V58:0.0027 & KI135:28.87 \\
\hline
\end{tabular}

Table 4: Number of observables, optimal design criterion, optimal cost, selected observables, and the minimum and maximum standard error and associated parameter for the parameter subset $\theta_{c}$. 


\section{Conclusion and future work}

In this paper we used an existing dynamical system plant model [16] to explore observation variable selection methods in experiments studying photosynthesis in matured plants. We performed sensitivity analysis to determine which sets of parameters were most affecting the metabolites expressed in the differential equation system, verified that the inverse problem may be performed on those selected parameters when full data of all 38 metabolites are available, and used both simple linear regression and optimal design criterion minimization to preliminarily select the most important subset of metabolites to measure in an experiment.

Implementation of experimental design techniques to inform plant growth studies has shown great potential; hence, this project has many directions in which it could continue. The long-term goals of this project are: (i) to improve the sampling time distribution and select which parameters should be estimated using a more advanced technique, (ii) to improve how the model accounts for environmental factors such as atmospheric $\mathrm{CO}_{2}$ and $\mathrm{O}_{2}$ concentrations, light level, and $\mathrm{H}_{2} \mathrm{O}$ availability and how the model includes changes in the plant over time, and (iii) perhaps investigate cohortand population-level models for use with destructive sampling techniques.

With even more time, we would consider the techniques described in [2] and also compare selections of different number of metabolites. Both of these problems would require numerous parameter estimations, which would take a

great amount of time. However, investigating these problems could potentially save industry partners a great amount of resources by reducing the number of sampling times, reducing the number of metabolites measured at each time a sample is taken, and informing researchers as to which parameters must be accurately estimated.

\section{Acknowledgments}

Much of this research was conducted during the 17th Annual Industrial Mathematical and Statistical Modeling Workshop for Graduate Students hosted by the Center for Research in Scientific Computation and the Department of Mathematics at N.C. State University with support for graduate student investigators provided by the National Science Foundation through the Statistical and Applied Mathematical Sciences Institute. 


\section{References}

[1] H. Akaike, A new look at the statistical model identification, IEEE Transactions on Automatic Control, 19 (1974), 716-723.

[2] H. T. Banks, A. Cinton-Arias and F. Kappel, Parameter selection methods in inverse problem formulations, Center for Research in Scientific Computation Technical Report CRSC-TR10-03, NCSU, November 2010; in Mathematical Model Development and Validation in Physiology: Application to the Cardiovascular and Respiratory Systems, Lecture Notes in Mathematics, Mathematical Biosciences Subseries, Springer-Verlag, 2011, to appear.

[3] H.T. Banks, M. Davidian, J.R. Samuels Jr. and K. L. Sutton, An Inverse Problem Statistical Methodology Summary, Center for Research in Scientific Computation Technical Report CRSC-TR08-01, NCSU, January 2008; Chapter 11 in Mathematical and Statistical Estimation Approaches in Epidemiology, (edited by Gerardo Chowell, Mac Hyman, Luis M.A Bettencourt and Carlos Castillo-Chavez), Springer, Berlin Heidelberg New York, 2009, 249-302.

[4] H.T. Banks, J.L. Davis, S.L. Ernstberger, S. Hu, E. Artimovich and A.K. Dhar, Experimental design and estimation of growth rate distributions in size-structured shrimp populations, Inverse Problems, 25, (2009), September, 095003 (28pp).

[5] H.T. Banks, J.L. Davis, S.L. Ernstberger, S. Hu, E. Artimovich, A.K. Dhar and C.L. Browdy, A comparison of probabilistic and stochastic formulations in modeling growth uncertainty and variability, Journal of Biological Dynamics, 3, (2009, 130-148.

[6] H.T. Banks, J.L. Davis and S. Hu, A computational comparison of alternatives to including uncertainty in structured population models, Center for Research in Scientific Computation Technical Report CRSC-TR09-14, June, 2009; in Three Decades of Progress in Systems and Control, (X. Hu, et al., eds.) Springer, New York, 2010, 19-33.

[7] H. T. Banks, S. Dediu, S. L. Ernstberger and F. Kappel, Generalized sensitivities and optimal experimental design, CRSC-TR08-12, September, 2008, (Revised), November, 2009; J. Inverse and Ill-posed Problems, 18 (2010), 2583.

[8] H.T. Banks, K. Holm and F. Kappel, Comparison of optimal design methods in inverse problems, Center for Research in Scientific Computation Technical Report CRSC-TR10-11, NCSU, July 2010; Inverse Problems, 27 (2011), 075002.

[9] H.T. Banks and S. Hu, Nonlinear stochastic markov processes and modeling uncertainty in populations, Center for Research in Scientific Computation Technical Report CRSC-TR11-02, NCSU, January, 2011; Math. Biosci. Engr., to appear.

[10] A. Cintrón-Arias, H. T. Banks, A. Capaldi and A. L. Lloyd, A sensitivity matrix based methodology for inverse problem formulation, CRSC Technical Report CRSC-TR09-09; J. Inverse and Ill-posed Problems, 17 (2009), 545 -564 .

[11] M. Davidian and D. Giltinan, Nonlinear Models for Repeated Measurement Data, Chapman \& Hall, London, 1998.

[12] G. D. Farquhar, S. von Caemmerer and J. A. Berry, A biochemical model of photosynthetic $\mathrm{CO}_{2}$ assimilation in leaves of $\mathrm{C}_{3}$ Species, Planta, 149 (1980), 78-90.

[13] J. W. Jones, G. Hoogenboom, C. H. Porter, K. J. Boote, W. D. Batchelor, L. A. Hunt, P. W. Wilkens, U. Singh, A. J. Gijsman and J. T. Ritchie, The DSSAT cropping system model, European J. Agronomy, 18 (2003), 235-265.

[14] G. A. F. Seber and C. J. Wild, Nonlinear Regression, Wiley, New York, 1989.

[15] P. Steduto, T. C. Hsiao, D. Raes and E. Fereres, AquaCrop - The FAO crop model to simulate yield response to water: I. Concepts and underlying principles, Agronomy Journal, 101 (2009) 426-437.

[16] X. G. Zhu, E. de Sturler and S.P. Long, Optimizing the distribution of resources between enzymes of carbon metabolism can dramatically increase photosynthetic rate: A numerical simulation using an evolutionary algorithm, Plant Physiology, 145 (2007), 513-526. 